\title{
ESTUDIO DEL COMPORTAMIENTO DEL FUEGO MEDIANTE SIMULACIÓN DE INCENDIOS FORESTALES EN CHILE*
}

\author{
Miguel Castillo Soto \\ Laboratorio de Incendios Forestales. \\ Facultad de Ciencias Forestales y Conservación de la Naturaleza \\ Universidad de Chile \\ migcasti@uchile.cl \\ Roberto Garfias Salinas \\ Departamento de Gestión Forestal y su Medio Ambiente. \\ Facultad de Ciencias Forestales y Conservación de la Naturaleza \\ Universidad de Chile \\ rgarfias@uchile.cl
}

\begin{abstract}
Resumen: Se efectúa una validación estadística del simulador de incendios forestales del Sistema KITRAL desarrollado por el Laboratorio de Incendios Forestales de la Universidad de Chile, mediante la recopilación de información procedente de incendios reales ocurridos en áreas rurales de la Octava Región de este país. Se definieron 16 variables relacionadas al tamaño y geometría de las áreas quemadas, las cuales fueron estudiadas mediante análisis multivariado. Se discute y analiza la similitud de los pronósticos con los datos observados en incendios reales. Las diferencias más importantes se producen en incendios de un tamaño inferior a 0,5 ha. En cambio, para incendios de tamaño superior a 5 ha, los niveles de similitud fluctuaron entre 85 y 94\%. En cuanto a la comparación de resultados específicos del comportamiento del fuego, en 15 de las 16 variables analizadas (el $93 \%$ de los casos), no se comprobaron diferencias significativas entre los valores simulados y los reales, corroborándose la validez en las estimaciones.
\end{abstract}

Palabras clave: KITRAL, simulación, comportamiento del fuego, incendio forestal.

* Recibido: 14-02-11. Aceptado: 07-07-11 


\begin{abstract}
We performed a statistical validation of forest fire simulator KITRAL System developed by the Forest Fire Laboratory at the University of Chile, by collecting information from real fires occurred in rural areas of the Eighth Region of the country. We defined 16 variables related to the size and geometry of the burned areas, which were studied by multivariate analysis. The paper discusses and analyzes the similarity of the predictions with the data observed in real fires. The most important differences are found in fires smaller than 0.5 ha, which corresponded to an expected result, due to poor reliability of the information in verysmall areas, where data such as topography, are generated by interpolation techniques. However, for fires larger than 5 ha, the levels of similarity ranged between 85 and 94\%. As for the comparison of specific results of fire behavior, in 15 of the 16 variables analyzed (93\% of cases), no significant differences were found between simulated and actual values, confirming the validity in the estimates.
\end{abstract}

Keywords: KITRAL, simulation, fire behavior, wildfire.

\title{
1. Introducción
}

El comportamiento del fuego, es un término que ha sido empleado para designar la forma de desarrollo del fuego y sus características físicas durante el transcurso del incendio. Corresponde al conjunto de efectos, principalmente de carácter físico y mecánico, que se observan en el ambiente afectado por la propagación del fuego (Julio, 2007). En su desarrollo, es posible estudiar algunos atributos, entre ellos la velocidad de propagación (Albini, 1976; Rothermel, 1972). Su cálculo permite la estimación de otros elementos que caracterizan al incendio (Julio, 2007). Conceptualmente, es el rango de incremento o expansión de un incendio, medido en forma lineal $(\mathrm{m} / \mathrm{min} ; \mathrm{m} / \mathrm{s} ; \mathrm{km} / \mathrm{h})$, o bien en términos de área $\left(\mathrm{m}^{2} / \mathrm{min} ; \mathrm{ha} / \mathrm{h}\right)$. Se expresa como resultado de la acción combinada de los factores del comportamiento, siendo los más relevantes la dirección y velocidad del viento, la inclinación y orientación de la pendiente, la temperatura y la cantidad, continuidad, distribución y calidad de la vegetación (Hepp y Vial, 1979). Los aspectos antes señalados fueron estudiados por Rothermel (1972), quien propuso expresiones matemáticas basadas esencialmente en fenómenos de transferencia del calor, y que sirvieron para modelar la propagación de incendios. En Chile se desarrolló un modelo de propagación de incendios en superficie (Julio, 2009), cuya fórmula y la discusión de sus resultados, corresponden al objetivo central de este artículo. Producto de la influencia de los factores que afectan al comportamiento del fuego, un incendio se propaga siguiendo 
patrones más o menos definidos. A esta conclusión llegó Anderson (1983), a base de múltiples observaciones realizadas, tanto en laboratorio como en terreno, demostrando que los incendios desarrollados bajo condiciones de viento constante, adquieren una forma que puede aproximarse a la elíptica o doblemente elíptica, con su semieje mayor elongado en la dirección del viento, y el semieje más corto, elongado en la dirección opuesta al avance del fuego. A su vez, producto de la propagación del fuego en múltiples direcciones, Van Wagner (1969), Albini (1976) y Forestry Canada Fire Danger Group (1992), distinguen los principales elementos geométricos que describen el tamaño y forma de un incendio forestal: lugar de inicio o foco, sección frontal o cabeza, sección posterior o cola, largo y ancho del incendio (figura 1). De este modo, la razón largo-ancho aumenta con el incremento de la velocidad de propagación de la porción delantera (Albini, 1976; Forestry Canada, 1992), siendo de mayor rapidez en presencia de pastizales que en otros modelos de combustible (Andrews, 1984; Julio, 2007). Este modelo ha sido estimado en términos de perímetro y área en distintos períodos de tiempo; a su vez, el ancho y largo del modelo elíptico es controlado principalmente por la velocidad y dirección del viento y la inclinación del terreno (Anderson, 1983; Brown y Davis, 1973; Rothermel, 1983).
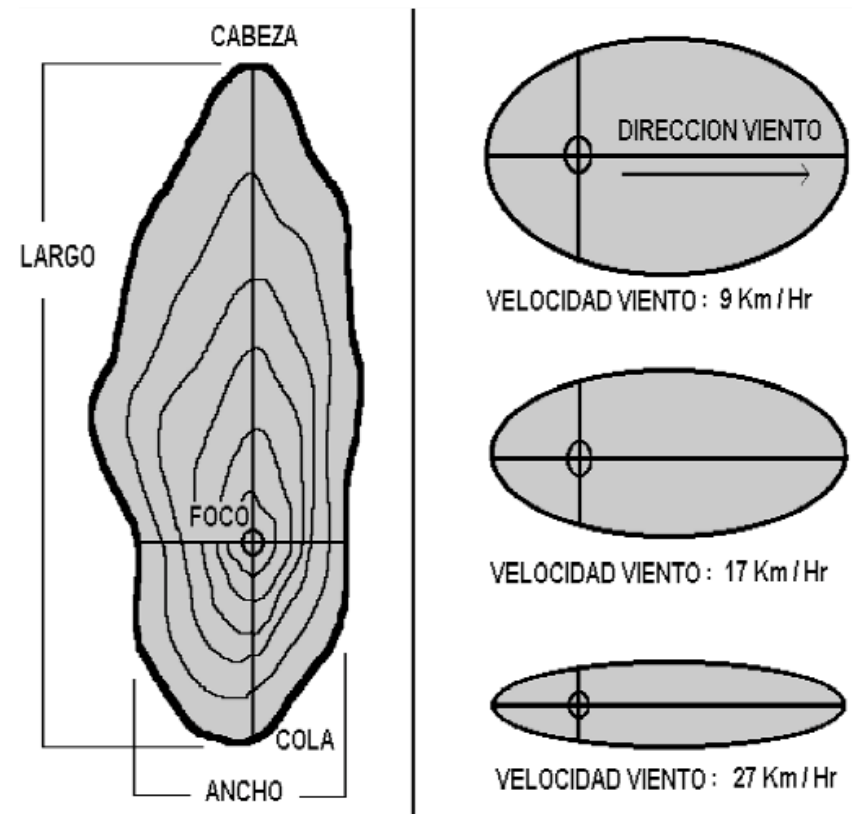

VELOCIDAD VIENTO: $9 \mathrm{Km} / \mathrm{Hr}$

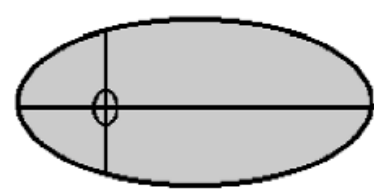

VELOCIDAD VIENTO: $17 \mathrm{Km} / \mathrm{Hr}$

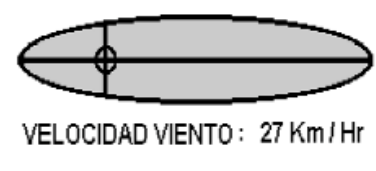

Figura 1. A la izquierda, se muestran los elementos geométricos básicos de un incendio. Las líneas concéntricas indican la propagación a distintas velocidades del viento, en cotas de isotiempo, modeladas en una simulación de prueba en KITRAL. En modelos más complejos el perímetro da lugar a expansiones o "dedos" que hacen más compleja la figura. A la derecha se muestra la variación en la elongación elíptica producto de la acción del viento. 
Para este patrón elíptico de propagación, Van Wagner (1969) estableció un modelo matemático que muestra en períodos sucesivos de tiempo, el crecimiento en superficie y perímetro afectados. Se señala la utilidad que presta este modelo en la resolución de problemas vinculados a la optimización de recursos asignados a la detección y control de incendios forestales. En síntesis, el modelo asume que después de un período inicial, la tasa de propagación en cada punto del perímetro varía continuamente desde un máximo, presente en la sección frontal (cabeza), hasta un mínimo en la sección posterior (cola) del incendio. Según Forestry Canada (1992), para que un fuego libre y de origen puntual adquiera la forma antes mencionada, deben presentarse condiciones de combustibles uniformes y continuos, topografía homogénea, viento constante y unidireccional, y que el fuego no sea afectado por actividades de supresión.

\section{2. Área de estudio}

El área de estudio corresponde a la Octava Región de Chile, cubriendo un área aproximada de $37.218 \mathrm{~km}^{2}$, caracterizada por una alta ocurrencia histórica de incendios forestales, especialmente en áreas cercanas a vías de comunicación y vegetación combustible. El clima es templado, con escasa oscilación térmica en la costa y temperaturas más elevadas hacia el interior de los valles.

El área de estudio señalada en la figura 2, se caracteriza por poseer una cubierta vegetal dominada por bosque y matorral esclerófilo, entremezclados con extensas plantaciones artificiales. En bosque esclerófilo sobresale el litre (Litbraea caustica), quillay (Quillaja saponaria), peumo (Cryptocarya alba), molle (Schinus latifolius) y belloto (Beilschmiedia miersii), entre otros. En bosque húmedo templado es posible encontrar roble (Nothofagus obliqua), raulí (Nothofagus alpina), coigüe (Nothofagus dombeyi), ruil (Nothofagus alessandrii) y hualo (Nothofagus glauca). Estas especies nativas coexisten con muchas otras de carácter exótico, y que han dominado estas latitudes por las favorables condiciones de clima y suelo. Respecto a plantaciones, actualmente la región presenta más de 939.000 hectáreas plantadas de pino (Pinus radiata), y numerosas variedades de especies de eucalipto.

\section{Materiales y métodos}

Por tratarse de una investigación orientada a la modelación del comportamiento del fuego en Chile, con una gran variedad de tipos de vegetación propias de este país, 


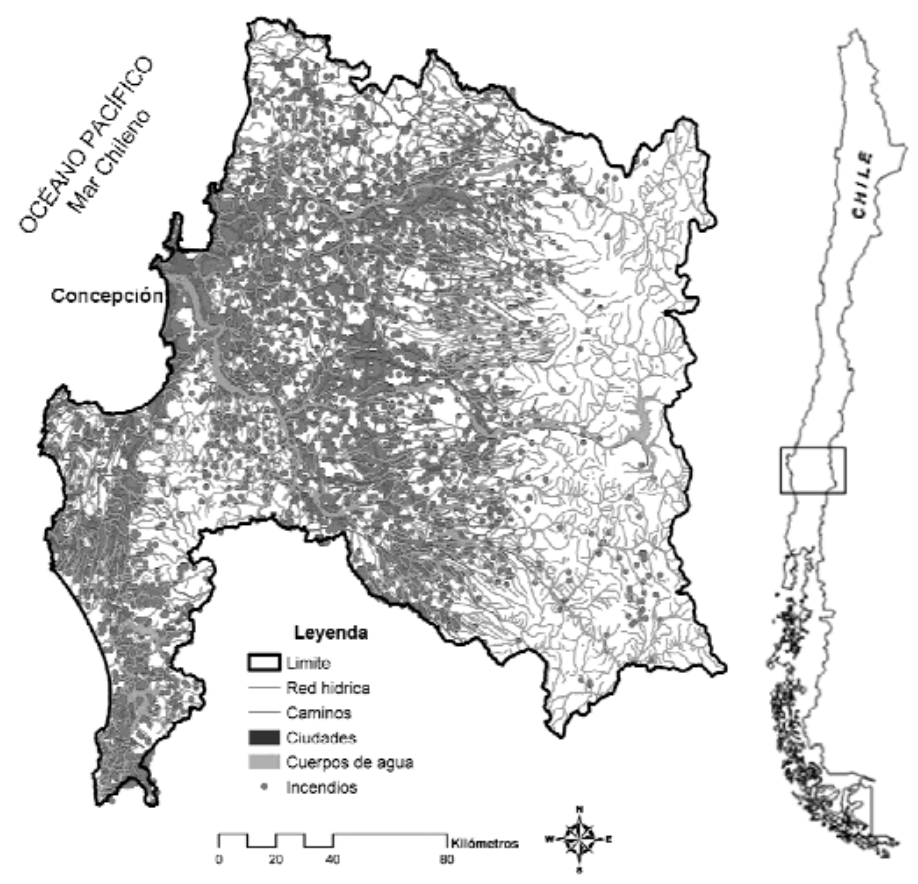

Figura 2. Área de estudio. Octava Región de Chile Centro-Sur. Las áreas demarcadas en rojo indican la alta frecuencia de incendios forestales ocurridos en los últimos 12 años (período 19982010). Se concentran generalmente a orilla de carreteras y en áreas de alto tránsito y flujo de turistas en épocas de verano. Escala: 1:1.500.000.

y cuyos parámetros físicos de propagación no pueden ser recreados en forma directa con otros simuladores en el mundo sin antes efectuar las correcciones y adaptaciones pertinentes, se utilizó el Simulador de Expansión de Incendios del Sistema KITRAL, cuya base conceptual y ecuaciones, proceden del Laboratorio de Incendios Forestales de la Universidad de Chile (Julio, 2007), fruto de la investigación de más de 45 años en el conocimiento de los incendios y características propias derivadas de los ambientes por los cuales se propagan.

Este sistema informático fue desarrollado en el año 1997, con el propósito de contribuir a elevar el nivel de eficiencia en la gestión contra incendios forestales en Chile, tanto en el sector público como privado. En términos específicos, se compone de cinco módulos generales que administran bases de datos de recursos terrestres y aéreos, junto a simuladores de pronóstico meteorológico, índice de riesgo y peligro de incendios forestales, asistencia al despacho de recursos para el combate, simulador de campos de vientos (VKITRAL), y el simulador de expansión de incendios. 
Todo lo anterior, desarrollado bajo una plataforma SIG que permite, entre otras operaciones, intercambiar datos con otras aplicaciones informáticas. KITRAL posee similares características a otros sistemas desarrollados tales como CARDIN, FlamMap, por nombrar algunos. Su originalidad destaca en la inclusión de un módulo estratégico de asistencia al despacho de recursos para el combate y el simulador meteorológico basado en índices de grado de peligro.

Se recopilaron antecedentes de 10 incendios reales localizados en el área señalada en la figura 2, mediante visitas a terreno en conjunto con las brigadas de control terrestre y aéreo. En cada evento, se tomaron datos referentes al lugar de inicio o foco del incendio, condiciones ambientales locales (temperatura del aire, humedad relativa, nubosidad, número de días desde la última lluvia y su intensidad, velocidad y dirección del viento (tabla 1), pendiente y exposición del terreno), variables del comportamiento del fuego (principalmente combustibles afectados por la propagación del fuego, longitud de llamas, y emisión de pavesas), antecedentes sobre expansión del incendio (apoyados en dibujos y planos de la zona afectada), los tiempos de inicio del incendio y contención; y finalmente, las superficies afectadas al arribo o llegada de la brigada y al control. En general, estos incendios corresponden a propagación en superficie, y fueron considerados como base muestral debido a las condiciones señaladas en la tabla 1 , propicias para recrear todo tipo de situaciones en terreno.

Tabla 1. Variables meteorológicas de incendios considerados para el estudio.

\begin{tabular}{ccccccc}
\hline \multirow{2}{*}{ Incendio } & $\begin{array}{c}\text { Tempera- Humedad } \\
\text { tura del } \\
\text { aire }\left({ }^{\circ} \mathrm{C}\right)\end{array}$ & $\begin{array}{c}\text { Relativa } \\
(\%)\end{array}$ & $\begin{array}{c}\text { Vel viento } \\
\text { (Km/h) }\end{array}$ & $\begin{array}{c}\text { Dirección } \\
\text { del viento } \\
\text { (grados) }\end{array}$ & $\begin{array}{c}\text { Combustible } \\
\text { dominante } \\
(1)\end{array}$ & $\begin{array}{c}\text { Humedad Comb. } \\
\text { finos y muertos } \\
(2)\end{array}$ \\
\hline 1 & 18 & 50 & 5 & 45 & MT01 & 9.94950 \\
2 & 23 & 50 & 14 & 300 & MT03 & 9.90040 \\
3 & 24 & 50 & 10 & 45 & PL09 - MT03 & 9.89058 \\
4 & 28 & 35 & 2 & 45 & MT03 & 5.92130 \\
5 & 13 & 60 & 5 & 45 & MT04 & 12.61860 \\
6 & 18 & 50 & 20 & 50 & PCH02 & 9.94950 \\
7 & 22 & 50 & 10 & 0 & PL05 & 9.91022 \\
8 & 21 & 45 & 2 & 45 & PCH01 & 8.61004 \\
9 & 20 & 50 & 18 & 40 & PCH02 & 9.92986 \\
10 & 24 & 46 & 2 & 45 & PCH02 - PL02 & 8.84258 \\
\hline
\end{tabular}

(1) Códigos de Modelos de Combustible definidos para el sistema KITRAL. (2) Valores obtenidos a base del cálculo del factor de Contenido de Humedad de la Vegetación Fina y Muerta. 
Se definieron las variables que serían analizadas al momento de efectuar las comparaciones. Al respecto, investigaciones publicadas por Anderson (1983), Albini (1976), Brown y Davis (1973), Forestry Canada (1992), Rothermel (1983), Van Wagner (1969), Vasconcelos y Guertin (1992), señalan que el Frente Principal de Avance del Fuego, la Sección Posterior o Cola del incendio, la Razón Largo/Ancho, la Superficie y Perímetro afectados, son los parámetros más empleados para caracterizar geométricamente el tamaño y forma del modelo de propagación de un incendio forestal. Sobre la base de estos antecedentes, se definió la tabla 2, y que corresponde a las variables consideradas para validar los incendios.

Tabla 2. Definición de variables de medición, correspondientes a los incendios evaluados.

\begin{tabular}{llc}
\hline Nombre & Descripción & Unidad \\
\hline SQ & Superficie Quemada & ha \\
PQ & Perímetro Quemado & $\mathrm{m}$ \\
IS & Incremento de Superficie Quemada & $\mathrm{ha} / \mathrm{min}$ \\
IP & Incremento de Perímetro Quemado & $\mathrm{m} / \mathrm{min}$ \\
DOCB, DOCL & Distancias Origen-Cabeza (DOCB), Origen-Cola (DOCL & $\mathrm{m}$ \\
RLA & Razón Largo-Ancho & - \\
DON, DONE, DONW, & Distancias en ocho Rumbos de Propagación (Norte=N; & \\
DOS, DOSE, DOSW, & Sur=S; Este=E; Oeste=W; Noreste=NE; Sureste=SE; & m \\
DOE, DOW & Noroeste= NW; Suroeste= SW). & grados \\
ORCB & Orientación de la Cabeza o Frente Principal de Avance \\
\hline
\end{tabular}

Con el apoyo de cartografía catastral escala 1:10,000 se dibujaron los incendios a escala para posteriormente traspasarlos a formato digital, previamente referenciados. Para la definición del entorno de simulación en KITRAL, se consideró como unidad mínima de información, píxeles de $25 \times 25$ metros. Otros antecedentes necesarios para el funcionamiento del sistema corresponden a la Resolución Temporal, referida a las cotas de isotiempo o lapsos de tiempos iguales de propagación en rumbos divergentes, expresados en bandas de colores, y, el Tiempo Máximo a Simular, que corresponde al tiempo límite al cual se desea efectuar la modelación. El método de simulación se basa en un esquema de estimación de costos de acceso, a través de una superficie rugosa, en el que el fuego (su propagación) corresponde al móvil que se desplaza, y la superficie conforma la resistencia al desplazamiento.

De esta manera, y considerando la interacción de todos los niveles de información integrados en la plataforma geográfica de KITRAL, es posible calcular y expresar espacialmente la velocidad de propagación, de acuerdo a la siguiente expresión:

$$
\mathrm{VP}=(\mathrm{Fmc})(\mathrm{Fch})(\mathrm{Fp}+\mathrm{Fv})
$$


Donde VP corresponde al valor $\left(\mathrm{ms}^{-1}\right)$ de velocidad de propagación lineal del fuego; Fmc es el factor atribuible al modelo de combustible contenido en la celdilla, Fch es el factor contenido de humedad de las partículas vegetales finas y muy finas, mientras que Fp y Fv son los factores que consideran el efecto de la pendiente y viento, respectivamente.

Sobre la base de esta fórmula de Velocidad de Propagación Lineal del Fuego propuesta en el Sistema KITRAL, es posible determinar el tiempo de propagación de fuego a partir de un punto de la zona inicialmente quemada (foco) hacia cualquiera de las celdas vecinas. El paso clave de la simulación consiste en determinar cuál de las celdas fronterizas será la más próxima en quemarse, transformándose en interna; lo anterior permite determinar en qué momento el fuego proveniente de esta celda alcanzará a sus vecinas que aún no se queman. Este esquema es iterativo, ampliándose la zona de simulación en cotas de isotiempo en cualquiera de los ocho rumbos de avance (figura 3).
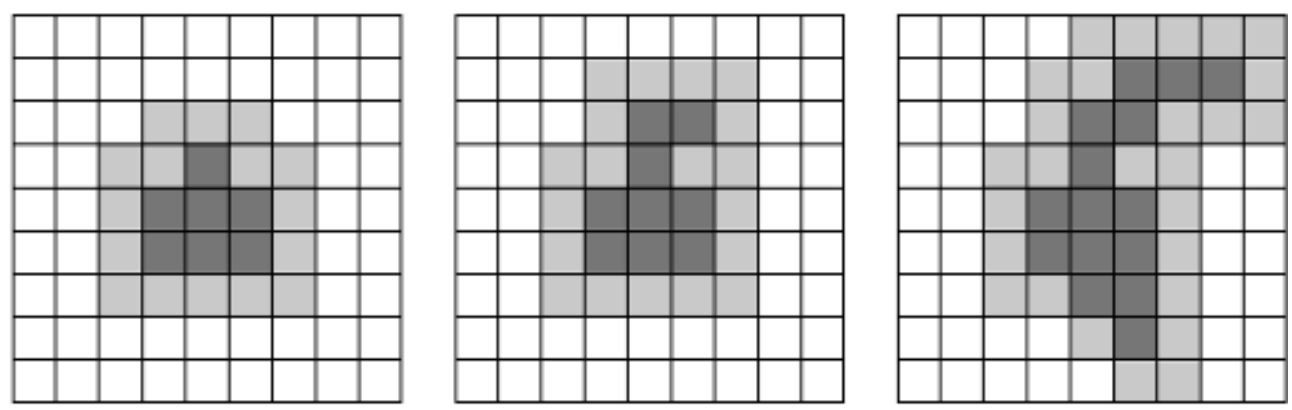

Figura 3. De izquierda a derecha, se muestra la ampliación de la zona afectada, en cotas de isotiempo, representadas aquí por distintas tonalidades de gris.

Sobre la base de este esquema de simulación, se aplicó un análisis multivariado al conjunto de datos, tomando ambos escenarios: los resultados generados de los incendios reales y los obtenidos por el simulador KITRAL para los mismos incendios evaluados. Para establecer una comparación estadística entre ambos escenarios, se analizó qué variables y en qué magnitud se expresan en los conceptos de "tamaño" y "forma" de un incendio forestal, a modo de establecer estadísticamente si existen similitudes entre ambos escenarios. El tamaño del incendio puede asumirse como crecimiento, representado en $n$ variables de medición. De este modo, al haber incremento de alguna variable, aumenta el tamaño. A su vez, la forma que el modelo de propagación adquiere, puede ser interpretada como el resultado de la propagación del fuego en distintos rumbos de avance. Así por ejemplo, una observación pareada 
que describe la superficie real y simulada de un incendio, puede arrojar valores similares, pero la forma resultante del incendio puede ser muy distinta. (Figura 4).

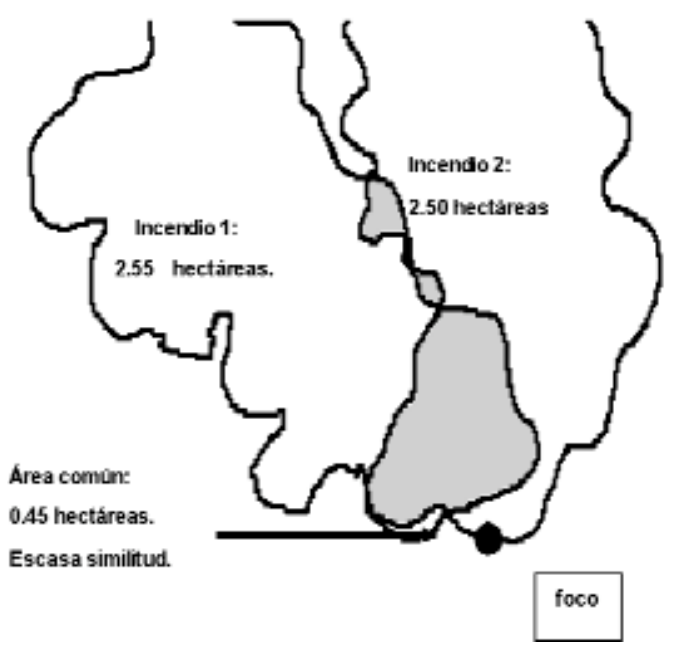

Incendio 1:

2.55 hectareas

Figura 4. Caso en que se muestran dos incendios aparentemente muy similares en tamaño, pero con frentes de avance (1) y formas (2) diferentes. En ambos casos: Incendio 1: situación real. Incendio 2: recreación de prueba en KITRAL, para ilustrar el concepto de "tamaño" y "forma".

\section{Resultados y discusión}

A base del análisis multivariado, se calculó la matriz de covarianza (Anexos 1 y 2), con el fin de expresar la dispersión de los datos reales y simulados respecto a la media. Por tratarse de dos escenarios a comparar, se generaron dos matrices utilizando los datos de las 16 variables señaladas en la tabla 2 , que sirvieron posteriormente para el cálculo de una transformación lineal que da origen a los valores y vectores propios, y los correspondientes valores de varianza explicada por cada componente Principal.

Examinando el aporte de cada uno de los componentes, se obtiene que en la matriz real, el primer componente absorbe el $89.69 \%$ de la variabilidad total, mientras que para la matriz obtenida de KITRAL, este valor para el primer componente asciende a un $94.36 \%$ de la variación total del conjunto de datos. Los valores y vectores propios utilizando la matriz de covarianza y la proporción de la variación explicada por cada componente principal, se muestran en los Anexos 3 y 4 . Mediante el uso del paquete estadístico SPSS se determinaron las transformaciones lineales nece- 
sarias para el cálculo de los coeficientes de correlación entre las variables y los componentes principales. De este paso, se determinó que las sucesivas simulaciones tomadas a base de los datos reales de incendios, reflejan que la primera combinación lineal del análisis multivariado, interpreta la característica del "tamaño" que adquiere un incendio forestal, en términos de longitud total del perímetro afectado a un tiempo $t$ o de control y, en menor magnitud, por el incremento de perímetro en tiempos iguales de propagación.

A su vez, los vectores propios para el $1^{\circ}$ componente simulado expresan de igual forma el concepto de "tamaño", es decir, con participación decreciente para las variables PQ e IP, seguidas en importancia por L y DOCB. Ello evidencia la correspondencia en la expresión de esta característica en ambas situaciones, para el $1^{\circ} \mathrm{com}-$ ponente principal. En las observaciones reales se observa una correspondencia entre el $2^{\circ}$ componente principal y la variable de largo del incendio L, seguida en orden de expresión por el perímetro PQ (0.5109 y 0.5070), mientras que las observaciones simuladas presentan como máxima correspondencia las variables de distancias DONW y DON (-0.4906 y -0.4650 respectivamente). Los vectores propios que conforman este $2^{\circ}$ componente, y los restantes quince componentes presentan, en ambos casos, valores positivos y negativos.

De las matrices de vectores propios a partir de la matriz de covarianzas, se muestra que en las restantes 16 combinaciones lineales, está la presencia de la expresión "forma", al existir una alta correlación entre los valores reales y simulados, para los 8 rumbos de propagación. En las observaciones reales, el 97.18\% de la variación total del perímetro PQ queda explicada por el primer componente, con un 2,78\% para el segundo. Esto significa que si se consideran los dos primeros componentes principales para esta variable, se habrá explicado el 99.96\% de la variación de PQ. A su vez, los resultados simulados indican que, tomando las dos primeras combinaciones lineales, se explica el $99.91 \%$ de la variación $\left(99.47 \%\right.$ para el $1^{\circ}$, y $0.44 \%$ para el $2^{\circ}$ ). Se aprecia la correspondencia entre los resultados reales y simulados en términos del aporte de las variables en los cinco primeros componentes principales (tabla 3). En ambas situaciones, el aporte de información que otorga la $8^{\circ}$ y $9^{\circ}$ combinación lineal será muy pequeño, siendo nulo en las otras 8 restantes. En el caso de IS e IP, estos valores fueron obtenidos a base del promedio obtenido en cada simulación, reflejados en los últimos 10 componentes.

La máxima proporción de varianza explicada para los valores del primer vector propio en el primer componente principal, queda reflejada en ambas situaciones al comparar estos valores con los obtenidos a partir del segundo componente. En ambos escenarios, se evidencia la alta proporción de la variación explicada por el primer componente (89.69\% real y $94.36 \%$ simulado). Sin embargo, existe una clara diferencia en el aporte de las variables IS, SQ, IP, PQ, DOCB, DON, DOS, DOE, DONE, DOSE, L, A y ORCB respecto a DOCL (1.85\% real y $0.42 \%$ simulado), DOW $(2.57 \%$ 
Tabla 3. Aporte residual en variación explicada (\%) en los últimos 10 componentes principales.

\begin{tabular}{lcc}
\hline Variable resultado & Real & Simulado \\
\hline Incremento de Superficie (IS) & 0,51 & 3,04 \\
Superficie Quemada (SQ) & 0,30 & 3,61 \\
Incremento de Perímetro (IP) & 0,03 & 0,01 \\
Perímetro Quemado (PQ) & 0,01 & 0,00 \\
Distancia Origen-Cabeza (DOCB) & 0,04 & 0,03 \\
Distancia Origen-Cola (DOCL) & 1,26 & 0,97 \\
Distancia Origen-Norte (DON) & 0,20 & 0,00 \\
Distancia Origen-Sur (DOS) & 0,21 & 1,64 \\
Distancia Origen-Este (DOE) & 1,95 & 0,57 \\
Distancia Origen-Oeste (DOW) & 1,14 & 1,82 \\
Distancia Origen-Noreste (DONE) & 0,07 & 0,03 \\
Distancia Origen-Noroeste (DONW) & 0,56 & 0,47 \\
Distancia Origen-Sureste (DOSE) & 7,17 & 0,55 \\
Distancia Origen-Suroeste (DOSW) & 6,37 & 3,71 \\
Largo (L) & 0,02 & 0,00 \\
Ancho (A) & 10,42 & 4,27 \\
Orientación de la Cabeza (ORCB) & 23,49 & 13,96 \\
\hline
\end{tabular}

real y 2.38 simulado) y DOSW (0.21\% real y $0.76 \%$ simulado), principalmente por la menor magnitud de las varianzas y de las medias. Esta situación, frecuente cuando se trabaja con variables que se expresan en distintas unidades y cuyos recorridos de valores difieren en uno o más órdenes de magnitud, puede influir de manera apreciable en los resultados. Por tal razón, es pertinente hacer uso de la matriz de correlación a partir de la matriz de covarianzas a fin de poder seleccionar un adecuado número de componentes que permitan establecer una concordancia con estos resultados.

Si se examinan las proporciones absolutas reales y simuladas, los cinco primeros componentes sintetizan porcentajes similares de varianza total $(93.26 \%$ real y $97.24 \%$ simulado), los siguientes cuatro lo hacen con valores de $6.73 \%$ real y $2.75 \%$ simulado, siendo los restantes ocho componentes nulos en cuanto a aporte en variación explicada, lo cual indica que, sin perder información, la dimensión del problema puede reducirse de diecisiete a nueve componentes, en ambos casos, siendo posible contener más del 90\% de toda la información en sólo cinco componentes principales. Si se utiliza la matriz de correlación, se incluirán los componentes cuyos valores propios sean mayores que 1 ( 1 es el promedio de todos los valores propios). Otro criterio, sugerido por Siegel (1956), tiende, a través de un método gráfico, incluir un alto número de componentes. Considerando lo anterior, se utilizarían los tres primeros, reales, cuyos valores fueron mayores que el promedio, es decir, superiores a 1 . En 
los valores propios simulados los cuatro primeros componentes superaron este promedio. A su vez, el método gráfico de Siegel representado para este estudio indicaron que en ambos escenarios (real y simulado), se puede considerar la selección hasta el tercer componente, a fin de alcanzar el $82.83 \%$ de la varianza explicada en las observaciones reales, y un $85.96 \%$ en las observaciones simuladas por el Sistema KITRAL (Figura 5).

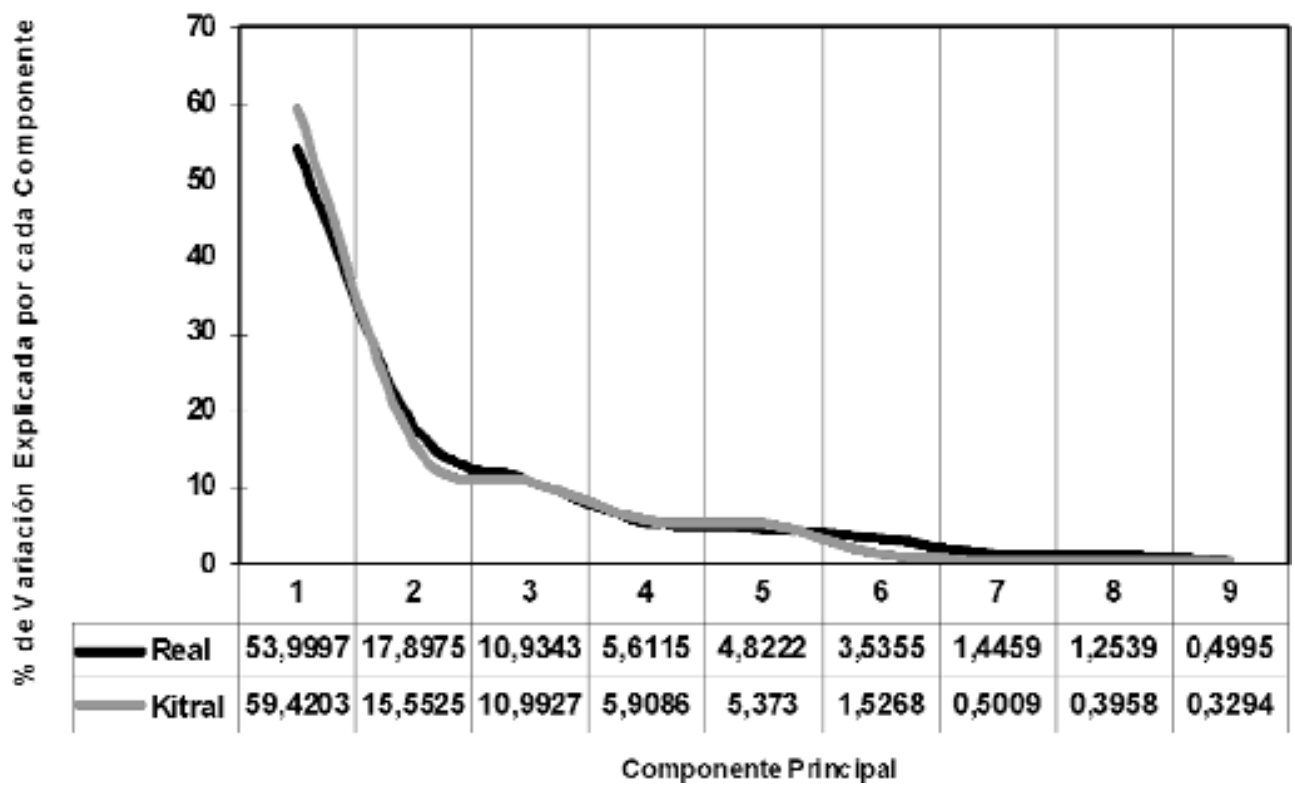

Figura 5. Variación explicada por cada componente. La similitud alcanzó a un $82 \%$ hasta la tercera combinación lineal para las observaciones reales, mientras que para los resultados del KITRAL, se obtuvo un valor cercano al $86 \%$.

Los coeficientes obtenidos de KITRAL indican la similitud respecto a los vectores reales, en los valores más altos para las variables de incrementos IS e IP, superficie SQ, perímetro PQ, distancia DONE, largo L y ancho A, representado en la proporción que ellas representan en el primer componente principal simulado. De este modo, los valores más elevados para el primer componente real, estarán asociados a incendios con tamaño superior al promedio, con un frente de avance claramente diferenciado de los ocho rumbos de propagación, una pequeña sección posterior o "cola" del incendio, escasa longitud de propagación hacia los rumbos W y SW, lo cual es lógico si se verifica que en la práctica los vientos dominantes de la zona en la cual se tomaron los datos meteorológicos tienen un predominio de las componentes EN, N y NW. 
A su vez, en el primer componente simulado se observa la similitud en la magnitud de DOW y ORCB, expresando esta combinación lineal, las características de propagación mencionadas en el caso real.

La expresión de tamaño en términos de IS, SQ, IP y PQ se expresa preferentemente en el primer componente, del mismo modo que el frente principal de avance. La similitud para las variables de distancia va siendo decreciente a partir de la tercera combinación lineal. Al observar los valores ligados al tercer y cuarto componente, las proporciones de varianza explicada son similares en ambos casos (10.93\% real y $10.99 \%$ simulado), pero la similitud en la expresión del tamaño y forma va disminuyendo al presentarse valores positivos y negativos muy distintos entre cada par de observaciones.

Si se consideran los dos primeros componentes principales, puede concluirse que las estimaciones realizadas por el simulador de expansión de incendios de KITRAL, en términos de la fórmula de velocidad de propagación, indican una alta similitud para incendios de gran tamaño, disminuyendo esta característica cuando los valores de perímetros y superficies son menores. A su vez, la forma que presenta el modelo de propagación en rumbos divergentes presenta mejores estimaciones para distancias Noreste y Norte, además del largo y ancho del contorno final de propagación. A medida que los incendios alcanzan un menor tamaño, la similitud para esta característica disminuye, siendo más certeras las estimaciones para las distancias DOCL, DOS y DOSW. A partir del tercer componente los resultados en las estimaciones tienden a ser variables, no presentándose una clara similitud en los resultados.

Puede graficarse la correlación de cada variable original con los dos primeros componentes principales. Para ello se extrae la raíz cuadrada de los valores de las proporciones de variación a partir de las matrices de correlación real y simulada, respetando el signo de los vectores propios. La correlación para el tercer y cuarto componente también son presentados gráficamente para observar las diferencias respecto a los dos primeros componentes. En una representación radial se han trazado las correlaciones reales y simuladas (Figura 6), con centro en el origen del par de coordenadas, lo cual permite identificar las variables cuya correlación con los componentes sea mayor (se acercan al círculo). Aquellas variables que se agrupan cerca del centro serán las que menos se correlacionan con los componentes principales. Como la proporción de la variación de cada variable explicada en los dos primeros componentes principales es la suma de sus correlaciones al cuadrado, las variables mejor explicadas se ubicarán cerca del círculo y las menos explicadas, cerca del origen de coordenadas. Estas correlaciones son graficadas de a pares (dos componentes sucesivas en cada caso), para cada variable resultado, resultando gráficamente lo siguiente: 


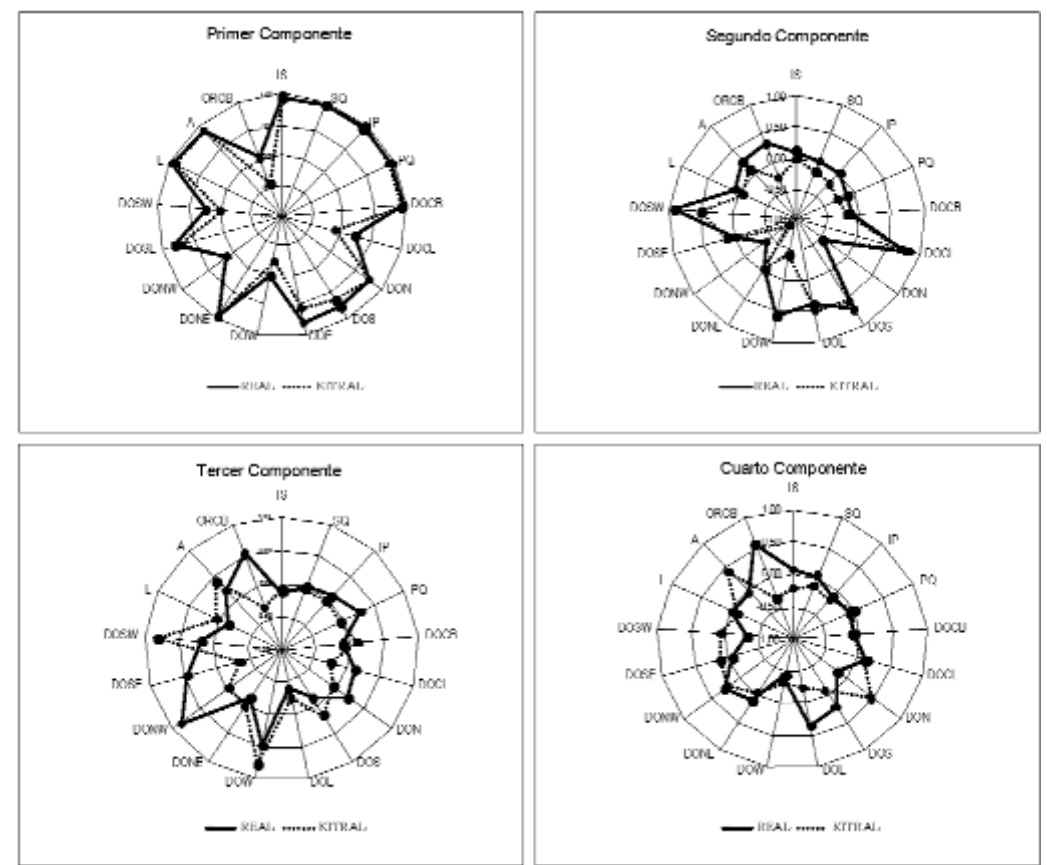

Figura 6. Representación conjunta de las variables resultado para cada componente principal. Las variables ubicadas a la periferia del círculo (valor 1) y cercanas al origen (valor -1), indican una alta correlación, a diferencia de las ubicadas cerca del valor cero.

Por lo anterior, la fórmula de propagación de KITRAL, tiende a otorgar resultados altamente similares para las variables IS, SQ, IP, PQ, en términos de tamaño del incendio, lo cual queda reflejado en el primer componente principal. La similitud en la forma expresada en las variables DOCB, DONE, L y A, con una menor contribución en la expresión de esta característica para las variables DON, DOS y DOSE. A su vez, el segundo componente expresa la variación en la forma, principalmente, en orden decreciente, por las variables DOCL, DOS, DOE y DOSW, asociado a incendios de menor tamaño y escasa propagación norte y noreste. Para el tercer y cuarto componente, no se aprecia una clara similitud en las correlaciones, por lo cual la certeza en las estimaciones tiende a disminuir, cuando se trata de incendios de tamaño pequeño.

De este análisis, fue posible estimar las similitudes en tamaño de las áreas quemadas, que por cierto constituye uno de los indicadores más frecuentemente utilizados por los especialistas al momento de utilizar un simulador de incendios forestales. Los valores de similitud antes señalados fueron traspasados a un segundo set de 
datos, aún más amplio ( $N=57$ incendios reales), para evaluar específicamente este parámetro. Con este porcentaje estimativo, y, con la base de datos adicional obtenida para los otros 47 incendios forestales reales (tabla 4) fue necesario determinar un parámetro indicador, que permitiese establecer entre qué valores o rangos de dicho parámetro, las estimaciones obtenidas del simulador de expansión se acercan o alejan de los resultados reales. Por disponer de antecedentes confiables de superficies afectadas para los 47 incendios antes mencionados, se optó tomar esta variable y adicionar las estimaciones obtenidas de los 10 incendios iniciales.

El promedio general para los 57 incendios reales evaluados, fue de 31,69 hectáreas, con una desviación de 105,73 hectáreas. El mínimo valor observado corresponde a 0.01 hectáreas, alcanzando a un máximo de 500 hectáreas. Por tal razón, para poder agrupar los valores en categorías de superficie, se ordenaron en forma ascendente y se dividieron en 5 rangos claramente distinguibles entre sí, cuidando que la desviación de cada rango fuera menor que el promedio del rango (tabla 4).

Tabla 4. Definición de Rangos de Superficies para 57 incendios evaluados y porcentajes de similitud obtenidos por KITRAL para los incendios simulados.

\begin{tabular}{lcccccc}
\hline & \multicolumn{5}{c}{ Rangos de Superficies, expresados en bectáreas } \\
\cline { 2 - 7 } & $0.05-0.50$ & $0.51-2.00$ & $2.01-5.00$ & $5.01-20.00$ & $20.01-60.00$ & $60.01-500.00$ \\
\hline Frecuencia & 15 & 26 & 5 & 4 & 3 & 4 \\
Promedio & 0.25 & 1.11 & 2.44 & 7.28 & 37.33 & 404.75 \\
Desv.estándar. & 0.22 & 0.53 & 0.54 & 1.30 & 20.03 & 87.07 \\
\% Similitud & 36.67 & 58.65 & 77.00 & 85.00 & 86.67 & 93.75 \\
\hline
\end{tabular}

Los promedios de superficies correspondientes a cada rango se ajustaron a distintos modelos estadísticos, resultando que el comportamiento de las predicciones se aproxima mejor a un modelo de tipo racional, cuya expresión es la siguiente:

$$
y=\frac{17.3818+143.1243 x}{1+1.5983 x-0.00018 x^{2}}
$$

Donde $\boldsymbol{x}$ representa el promedio de superficie real de cada rango, e $\boldsymbol{y}$, el indicador del porcentaje de similitud en las estimaciones obtenidas por KITRAL, para cada rango. 


\section{Conclusiones}

La fórmula de Propagación Lineal del Fuego (basada en la interrelación de los factores Modelo de Combustible, Contenido de Humedad de la Vegetación Fina, Velocidad del Viento y Pendiente), como así también su aplicación en la definición del modelo espacial del incendio (en ocho rumbos divergentes y tiempos de acceso desde un punto afectado por el fuego hacia los píxeles vecinos), demostraron una alta confiabilidad en la recreación de incendios reales.

Es necesario mencionar, que los valores de las variables de entrada, en especial los referidos a la humedad relativa y velocidad y dirección del viento, requieren ser lo más preciso posibles, para así eliminar la posibilidad de sesgo en la simulación atribuible al ingreso de datos. Es de particular importancia hacer énfasis que, si se desea obtener una buena simulación, es necesario contar con información confiable respecto a las condiciones ambientales del sector local en que se encuentra ubicado el incendio, como así también, en la ubicación precisa del lugar de inicio del incendio.

Un factor esencial que define el modelo espacial de propagación del fuego, es el viento. Al respecto, la información de vientos ingresada al sistema KITRAL, para los incendios registrados en la última temporada, procedió fundamentalmente de los resultados proporcionados por el simulador de Campos de Vientos VKITRAL, según la información diaria reportada por las estaciones meteorológicas. VKITRAL, frente a una solicitud de vientos, entregó en forma automática resultados razonables en la mayoría de los casos. En ocasiones, fue necesario corroborar y apoyar las estimaciones con información local, cuando fue posible obtener dicha información e ingresarla en forma manual al sistema.

A mayores superficies, los resultados de KITRAL tienden a ser más confiables. Por el contrario, en incendios pequeños, por ejemplo, una diferencia de 0,05 hectáreas en la estimación de una superficie real de 0,1 hectáreas, es muy significativa pues se sub-estima en un 100\%. A su vez, una sobre estimación de 26 hectáreas para un incendio con superficie real de 290 hectáreas, genera menores diferencias (9\% de sobre estimación), dada la magnitud de las superficies. 


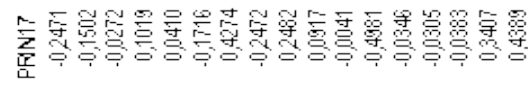

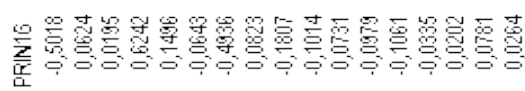

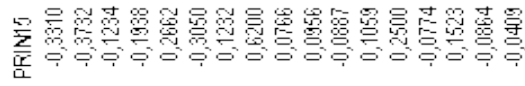

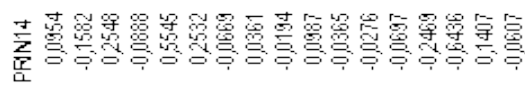

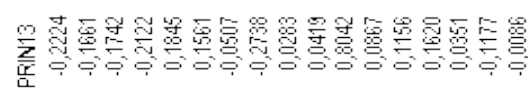

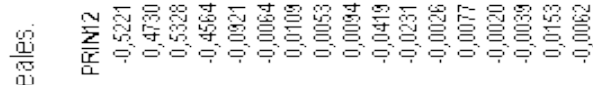

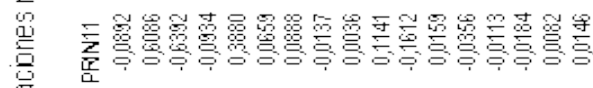

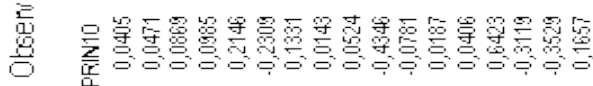

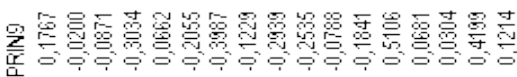

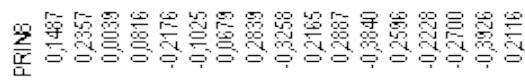

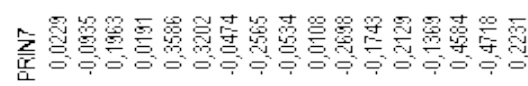

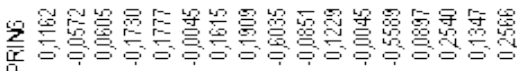

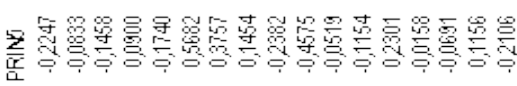

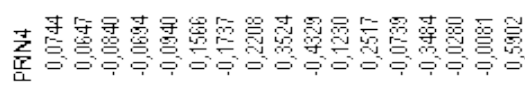

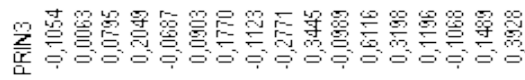

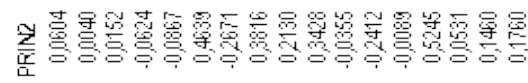

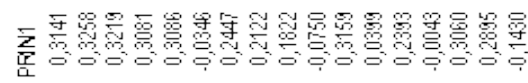

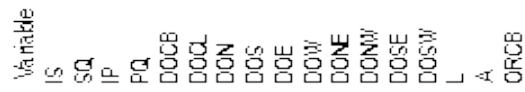

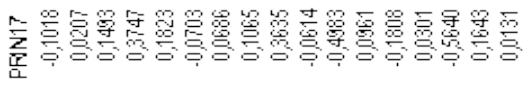

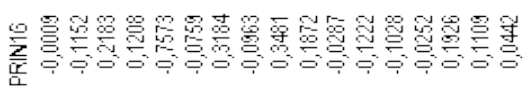

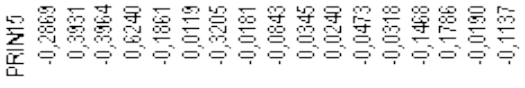

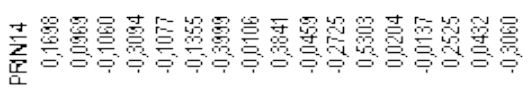

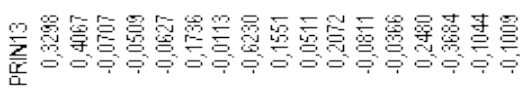

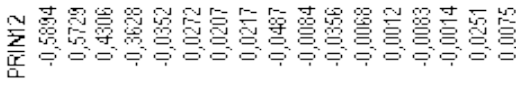

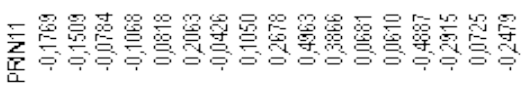

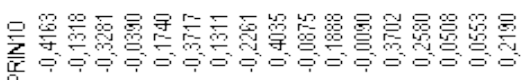

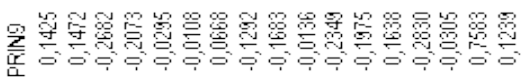

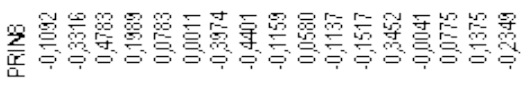

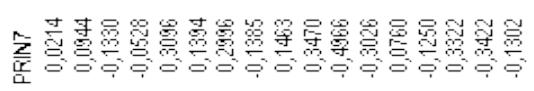

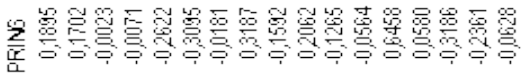

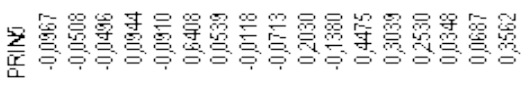

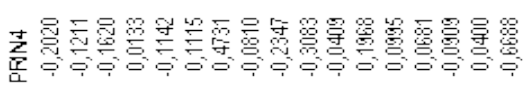

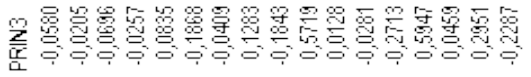

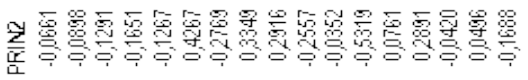

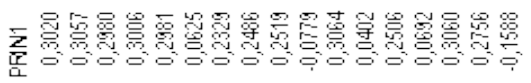

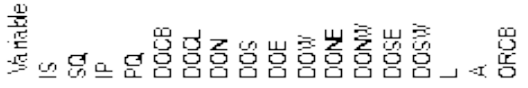




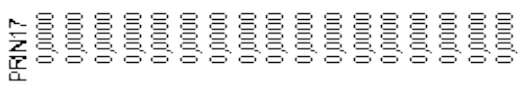

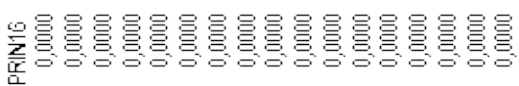

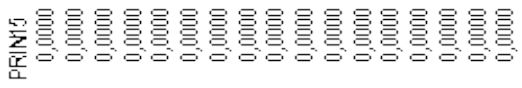

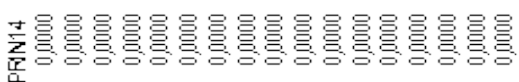

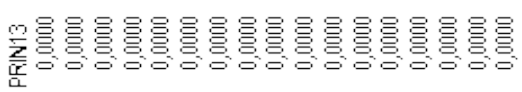

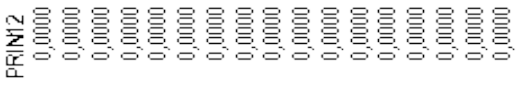

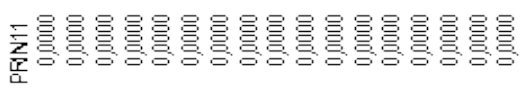

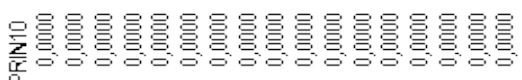

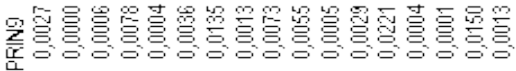

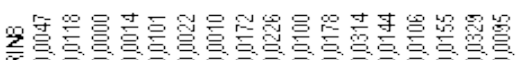

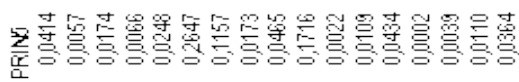

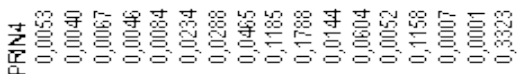

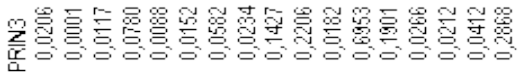

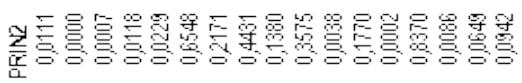

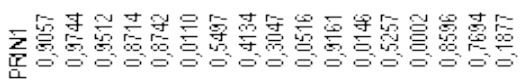

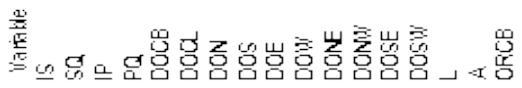

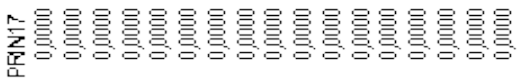

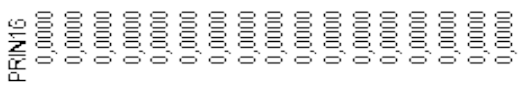

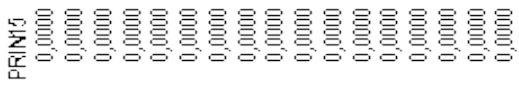

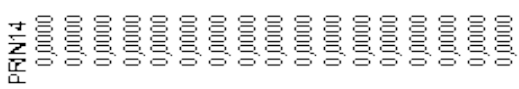

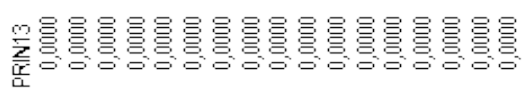

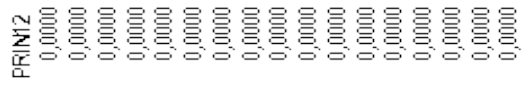

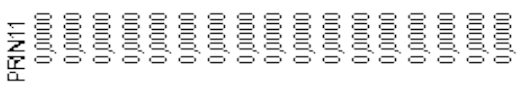

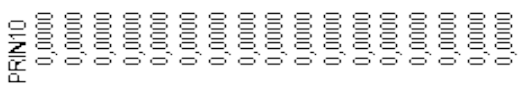

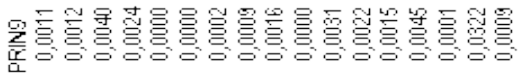

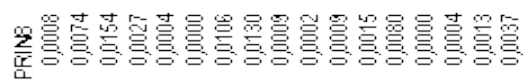

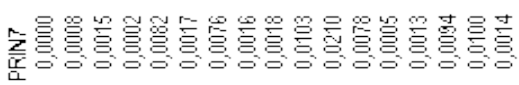

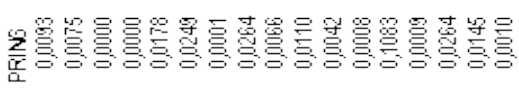

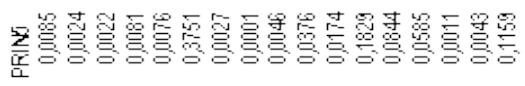

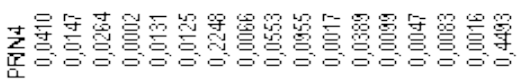

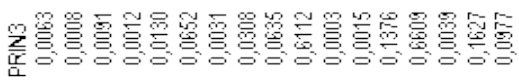

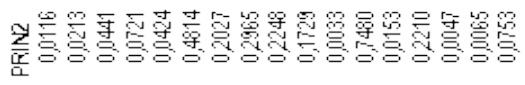

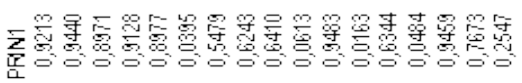

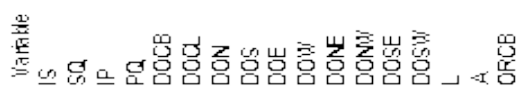




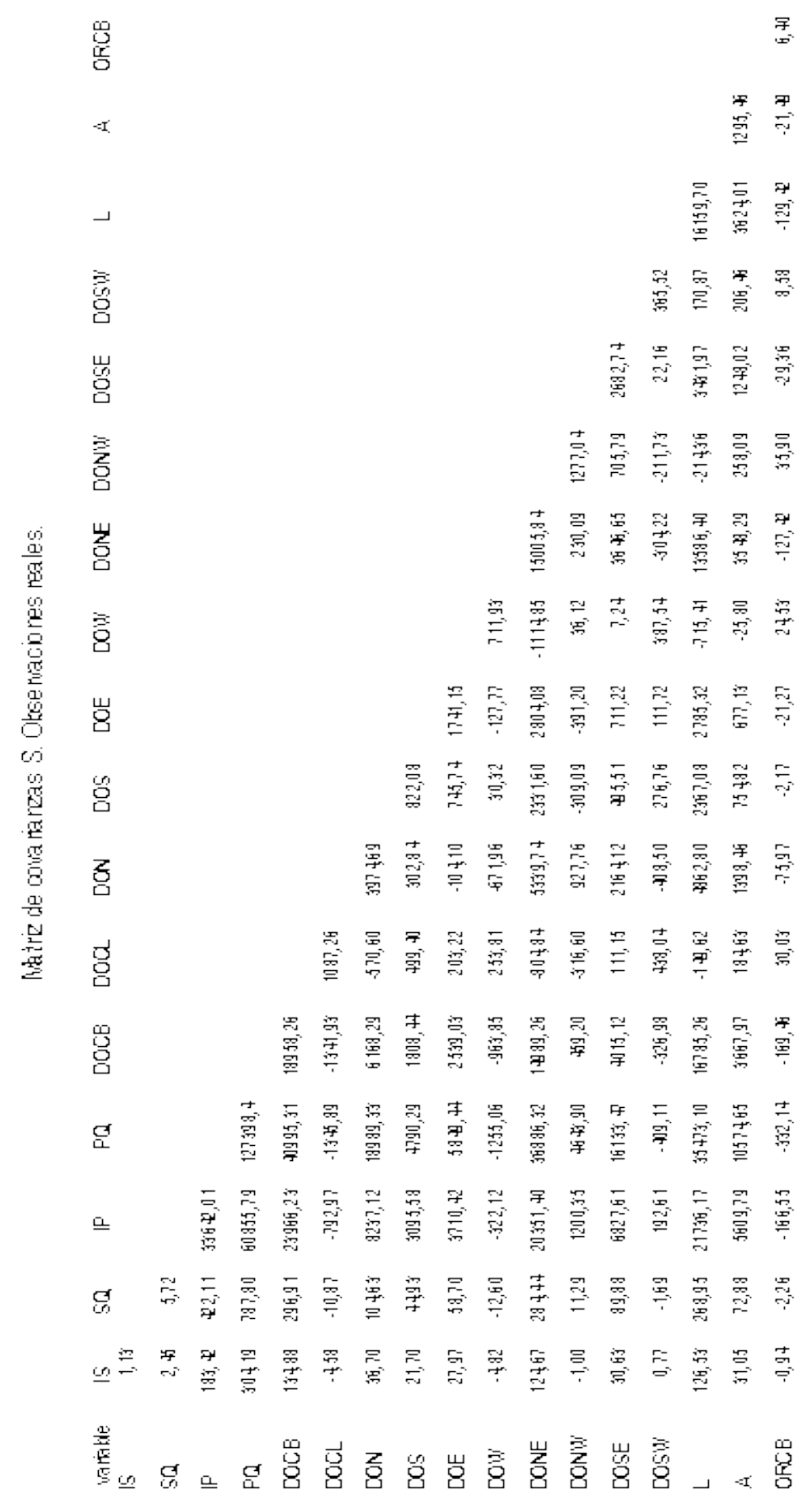




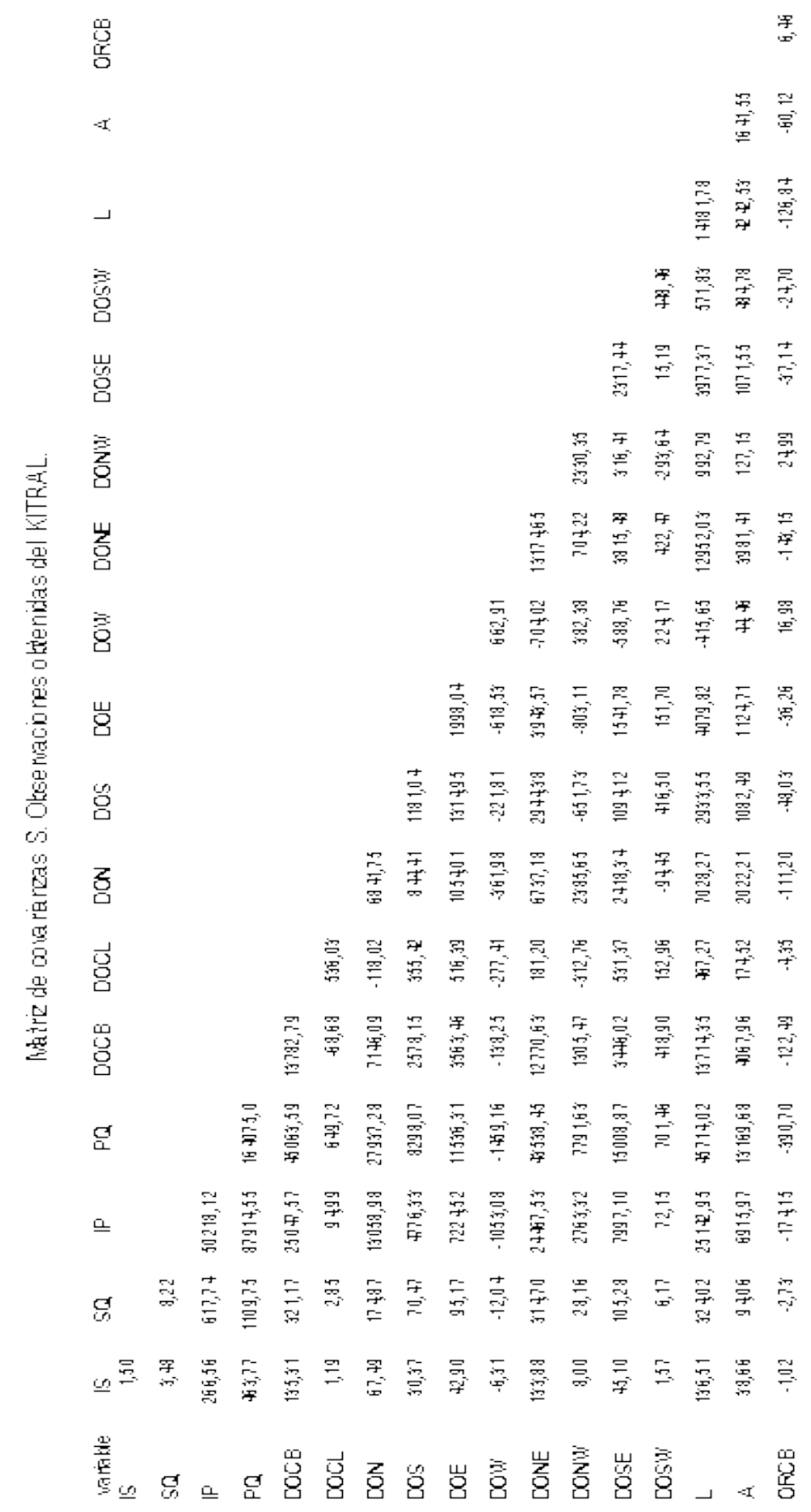




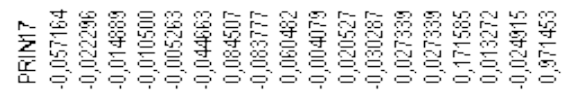

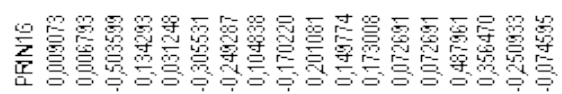

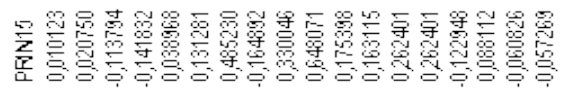

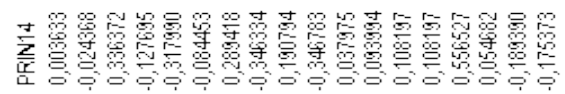

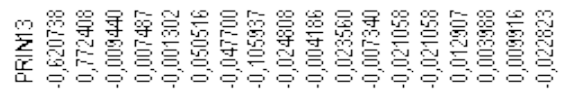

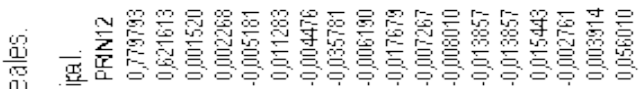

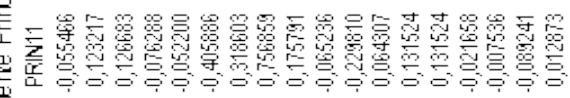

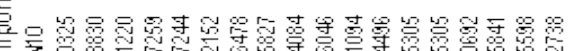

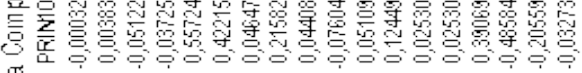
菅

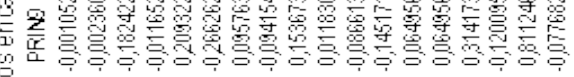

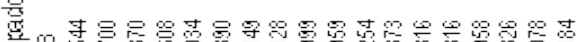

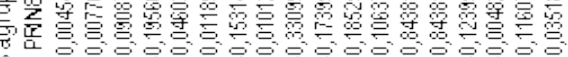

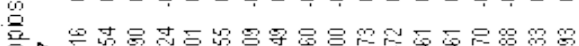

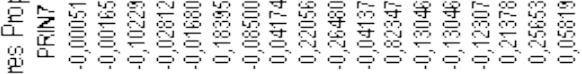

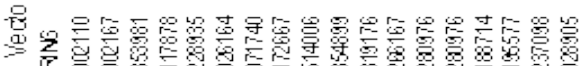

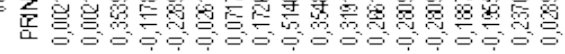

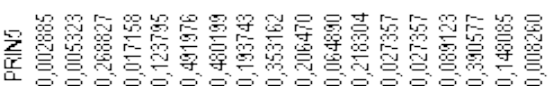

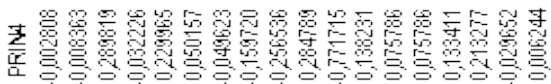

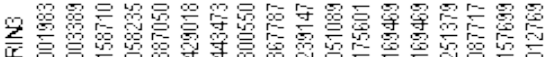

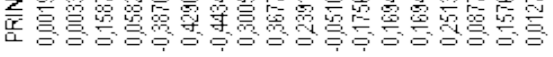

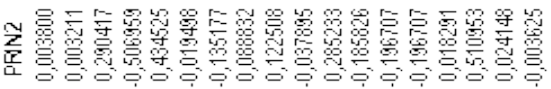

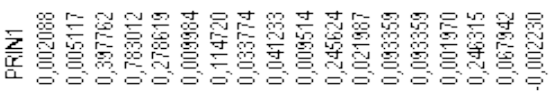

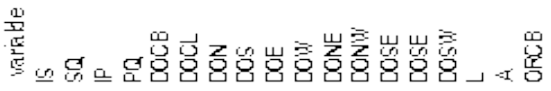

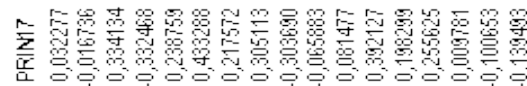

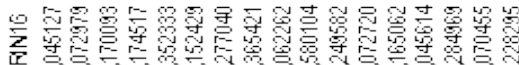

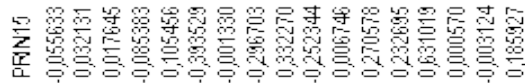

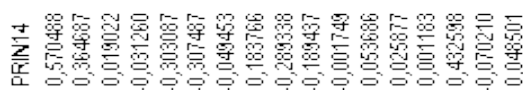

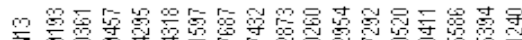

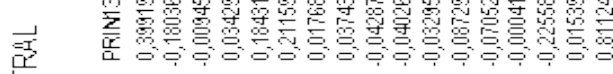

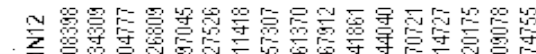

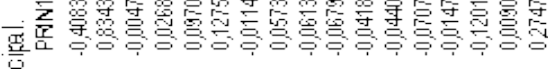

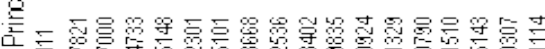

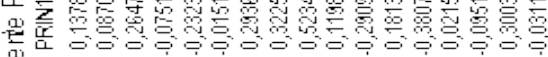

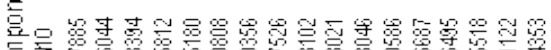

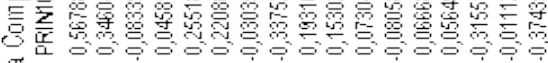

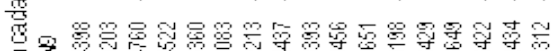

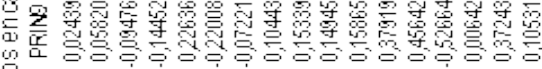

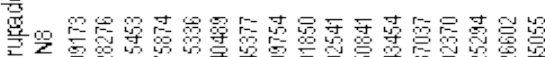

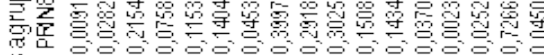

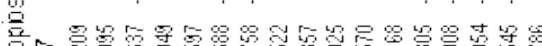

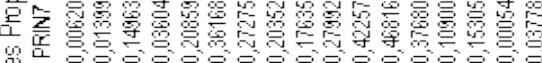

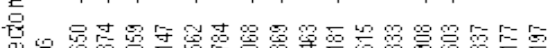

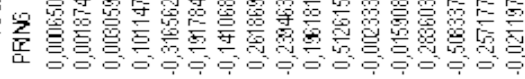

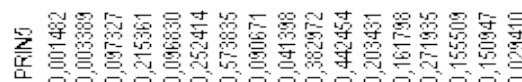

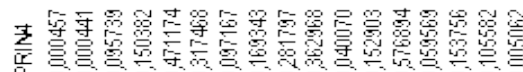

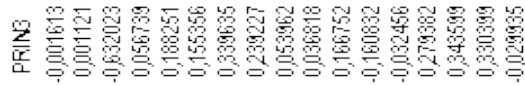

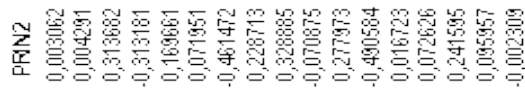

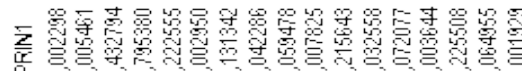

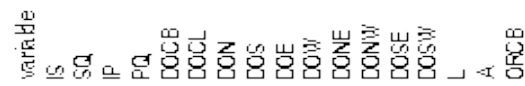




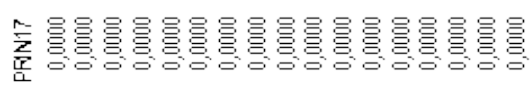

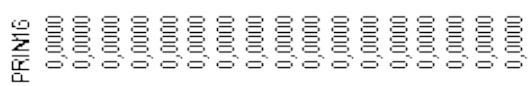

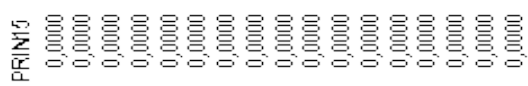

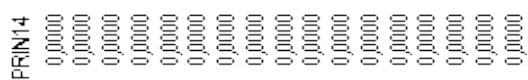

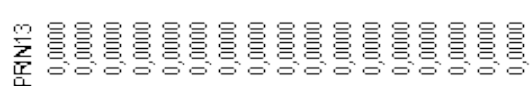

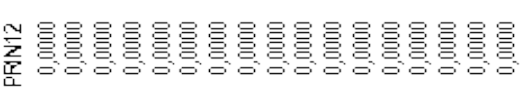

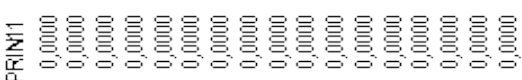

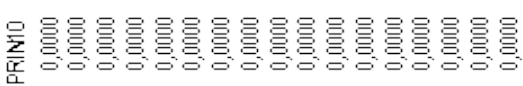

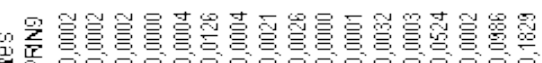

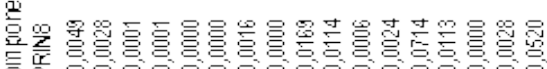

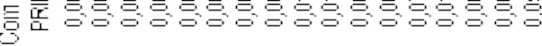

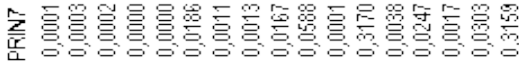

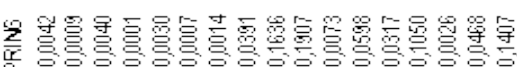

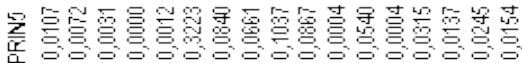

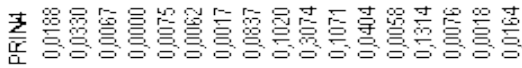

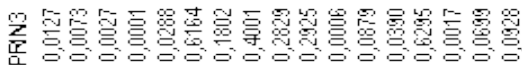

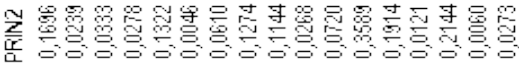

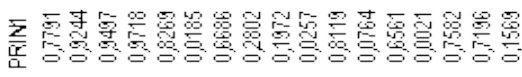
量

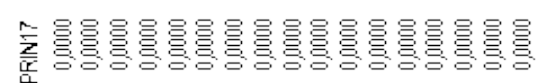

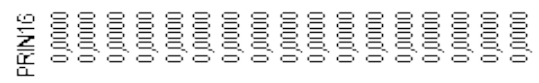

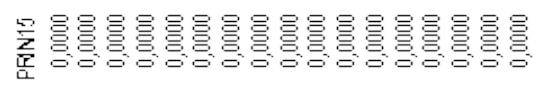

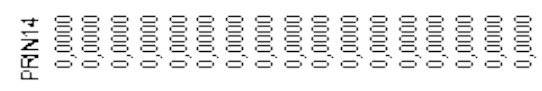

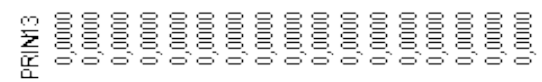

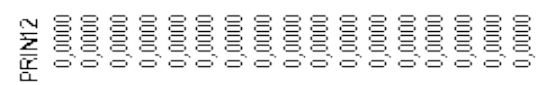

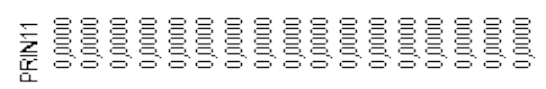

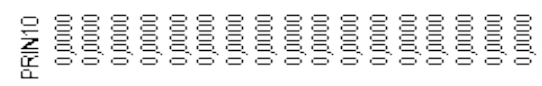

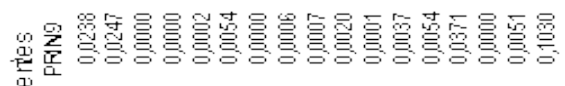

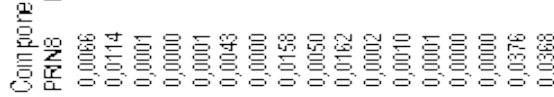

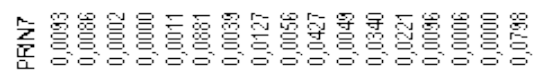

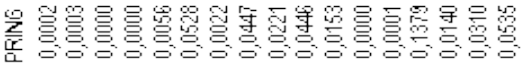

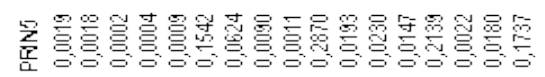

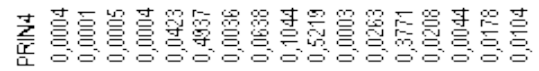

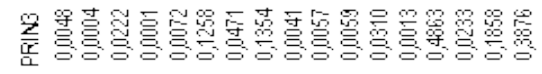

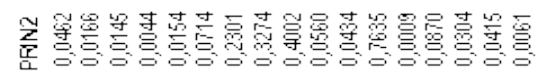

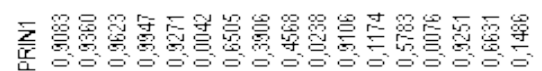
尊 


\section{Agradecimientos}

Proyectos Fondecyt 1095048 y Fondef FI-13.

\section{Referencias}

Anderson, H. (1983) Predicting wind-driven wild land fire size and shape. Ogden, USDA Forest Service, RES. Pap. INT-305.

Albini, F. (1976) Estimating wildfire behavior and effects. Ogden, USDA Forest Service. Gen.Techn.Rep. INT-30.

Andrews, P. (1984) BEHAVE: Fire behavior and fuel modeling system. Burn subsystem. Working Draft, $60 \mathrm{p}$.

Brown, A. y Davis, K. (1973) Forest fire Control and use. $2^{\text {nd }}$ ed, New York, Mc.Graw-Hill.

Forestry Canada Fire Danger Group (1992) Development and structure of the Canadian Forest Fire Behaviour Prediction System. Ottawa, Forestry Canada Information Report ST-X-3.

Julio, G. (2007) Apuntes del curso: Control de incendios forestales. Universidad de Chile. Depto. Manejo Recursos Forestales.

Julio, G. (2009) Formulación de lineamientos políticos y estratégicos para la protección contra incendios forestales en Chile. Tesis
Doctoral, Universidad de Córdoba, España.

Hepp, A. y Vial, E. (1979) Estudio del comportamiento del fuego en los incendios forestales de la VRegión. Tesis, Universidad de Chile.

Rothermel, R. (1972) Concepts in fire modeling. National Fire Training Center. Marana Air Park, 21p.

Rothermel, R. (1983). How to predict the spread and intensity of forest and range fires. Ogden, USDA Forest Service. Gen.Techn.Rep. INT-143, Ogden.

Siegel, S. (1956) Nonparametric statistics for the behavioural sciences. New York, McGraw-Hill.

Van Wagner, C. (1969) A simple fire growth model. Forestry Chronicle. 45(2): 103-104.

Vasconcelos, M. y Guertin, D. (1992) FIREMAP, Simulation of fire growth with a Geographic Information System. International Journal of Wildland Fire, 2: 87-96. 